\title{
Cryptosporidium spp. e Giardia spp. em carnívoros silvestres do Estado do Pará - Brasil*
}

\section{(Cryptosporidium spp. and Giardia spp. in wild carnivorous animals the State of Pará - Brazil)}

\author{
Darlene Kássia Saraiva Queiroz Pantoja, ${ }^{* *}$ Mônica Cristina de Moraes Silva, ${ }^{* * *}$ Heyde Araújo Tavares, ${ }^{* * *}$ \\ Marcella Katheryne Marques Bernal, ${ }^{* *}$ Dayane Novais da Luz Brito, ${ }^{* * *}$ Thais Lopes Chaves Santos, ${ }^{* * *}$ \\ Washington Luiz Assunção Pereira**
}

\begin{abstract}
Resumo
O presente estudo investigou a ocorrência de Cryptosporidium spp. e Giardia spp. em diferentes espécimes silvestres da ordem Carnívora de vida livre e de cativeiro procedentes de municípios do Estado do Pará. Coletou-se amostras fecais de 37 animais distintos (quatro de vida livre e 33 de cativeiro). Para pesquisa de Cryptosporidium spp. e Giardia spp. foram utilizados métodos microscópicos (direto e Kinyoun) e imunológico (RIDA ${ }^{\circledR}$ QUICK Cryptosporidium/Giardia/Entamoeba Combi - N1722). Do total de amostras, 24,32\% (9/37) foram positivas, correspondendo a 5,4\% (2/37) para Cryptosporidium spp. e 18,91\% (7/37) para Giardia spp., respectivamente. Nenhum animal apresentou infecção concomitante para os agentes. Cryptosporidium spp. e Giardia spp., são protozoários zoonóticos que representam um emergente problema de saúde pública. Esses parasitos podem apresentar elevada frequência em regiões em que as condições de saneamento básico são precárias, promovendo surtos de diarreia em animais domésticos, silvestres e no homen. Mamíferos silvestres, como os carnívoros, são susceptíveis à contaminação por enteroparasitas presentes tanto no habitat natural como em cativeiro. Portanto, a pesquisa comprova a presença desses protozoários em carnívoros silvestres, tanto mantidos em criatórios como nos de vida livre no Estado do Pará, considerando-se que esses animais podem atuar como fontes de infecção para o homem, para outros animais e para o meio ambiente.
\end{abstract}

Palavras-Chave: criptosporidiose, giardiase, mamíferos silvestres, protozoários, Pará.

\begin{abstract}
The presente survey has had the purpose to investigate the occurrance of Cryptosporidium spp. and Giardia spp. in free and under captivity carnivorous wild animals, from several counties in the State of Pará. Samples of feces from 37 distinct animals (four in their natural habitat and 33 raised in captivity). For the research of Cryptosporidium spp. and Giardia spp. microscopic immunological, direct and Kinyoun methods were used (RIDA ${ }^{\circledR}$ QUICK Cryptosporidium/Giardia/Entamoeba Combi - N1722). The samples gathered from wild animals have resulted in $24,32 \%$ of positive infecction on the rate of (9/37), being. 5,4\% (2/37) positive to Cryptosporidium spp. and 18,91\% (7/37) positive to Giardia spp., what shows that no amimals had both infections at the same time. Cryptosporidium spp. and Giardia spp., are zoonotic enteroparasites that have been taking place as an emmerging problem to public health. Theese species of protozoa may reach high levels of frequency in regions where the basic sanitation conditions are precarious, promoting outbraks of diarrhea to men, wild and domestic animals. Wild mammals, as the carnivorous, are susceptible to contamination by enteroparasites, being present at their natural habitat or captivity. So, the reserach strenghtens the real presence of these protozoas in wild carnivorous in both conditions of life, free or under captivity, in the State of Pará, making us consider the possibility that the cited animals may be natural reservoirs for infections, not only to men but to other animals and also to environment.
\end{abstract}

Keywords: Cryptosporidium spp., Giardia spp., mammals, protozoal, Pará.

\section{Introdução}

Estudos sobre a ocorrência de parasitos em felinos silvestres apresentam grande importância, pois estes podem se constituir problemas zoonóticos em áreas florestais e/ou naquelas destinadas à visitação de pessoas (AIRES et al., 2008). Podem, também, contribuir para um melhor entendimento da influência e intensidade que as mudanças antrópicas promovem nos ambientes selvagens, incluindo informações adicionais sobre a fauna parasitária de animais silvestres (HOLSBACK et al., 2013).

A ocorrência de mudanças ambientais exerce grande influência na proliferação e no surgimento de doenças parasitárias

\footnotetext{
${ }^{*}$ Recebido em 2 de julho de 2019 e aceito em 21 de outubro de 2019.

**Universidade Federal Rural da Amazônia, Laboratório de Patologia Animal, Belém, Pará

***Instituto Evandro Chagas, Seção de Parasitologia, Anannindeua, Pará.

Autor para correspondência: Marcella Katheryne Marques Bernal. mkbernalfh@gmail.com.
} 
zoonóticas, entre elas a criptosporidiose e a giardíase, sendo que as mudanças promovidas por fenômenos naturais ou produzidas pela intervenção antrópica, podem levar a alterações ao equilíbrio ecológico e, consequentemente, a ocorrência de agentes patogênicos em seus hospedeiros silvestres e vetores (LALLO et al., 2009). Nesse contexto, a saúde pública vem evidenciando uma crescente preocupação da atuação de animais silvestres como portadores e veiculadores de agentes zoonóticos (FANFA et al., 2011).

Espécies de carnívoros silvestres podem ser acometidas por vários gêneros de parasitos intestinais dos quais podem servir apenas como reservatórios ou desenvolverem a doença clínica (FIORELLO et al., 2005). WHITEMAN et al. (2008), alertam que a presença de carnívoros domésticos em unidades de conservação tem se tornado uma preocupação na região amazônica brasileira, ao qual evidencia-se que a introdução desses animais pode oferecer riscos à saniadade de carnívoros e outros mamíferos silvestres em áreas protegidas.

Parasitos do gênero Cryptosporidium são gastroentéricos considerados oportunistas, capazes de infectar praticamente todas as espécies de vertebrados (BRIGGS et al., 2014). A criptosporidiose pode ser causada por diferentes espécies e subtipos de Cryptosporidium spp., afetando diversos hospedeiros cujos sinais clínicos variam de acordo com a idade, estado sanitário, espécie e dose infectante do parasito (XIAO; FAYER, 2008). Em mamíferos silvestres a carga parasitária por Cryptosporidium spp. tende a ser menor quando comparada aos valores encontrados em mamíferos domésticos (FAYER; XIAO, 2008).

A transmissão do Cryptosporidium spp. é predominantemente fecal-oral, sendo a veiculação hídrica a principal via de disseminação do agente (ONICHANDRAN et al., 2014). Thompson et al. (2010), destacam que a diversidade de Cryptosporidium spp. encontrado na vida selvagem merece estudo adicional em termos de ecologia, evolução, biologia e impacto potencial sobre o saúde desses animais. O conhecimento da cadeia de transmissão do parasito pode proporcionar uma oportunidade para verificar como esses agentes alcançam novos hospedeiros suscetíveis, assim, em relação aos fatores epidemiológicos, os animais silvestres podem ter um papel extremamente importante na transmissão de zoonoses, tanto em cativeiro quanto em vida livre (MARVULO, 2007).

Já com relação a giardíase, a incidência e a prevalência em humanos e animais têm sido relatada em todo o mundo, variando consideravelmente entre as populações e localizações geográficas estudadas (LINDSAY; ZAJAC, 2009). Os animais servem como fontes de infecção e podem eliminar cistos pelas fezes por meses ou anos (LALLO et al., 2003). O número de cistos ingeridos pelo hospedeiro desempenha um papel de destaque na patogênese da giardíase (LINDSAY; ZAJAC, 2009). Os animais adultos são portadores assintomáticos na grande maioria dos casos, o que contribui para a eliminação de cistos do parasita no meio ambiente, podendo contaminar outros animais e o homem (BARR; BOWMAN, 2010).

A grande maioria dos dados obtidos sobre Giardia spp. em mamíferos silvestres, origina-se de estudos feitos para avaliar o potencial destes animais como reservatórios de doença para humanos e para o gado (APPELBEE et al., 2005).
De acordo com Soares et al. (2008) o potencial zoonótico de protozoários a partir de hospedeiros silvestres é ainda pouco estudado no Brasil. Portanto, estudos que avaliem a prevalência destes agentes em animais silvestres cativos e de vida livre são necessários para o entendimento da dinâmica de transmissão destes agentes.

Nesse sentido, buscou-se com o presente trabalho, investigar a ocorrência de Cryptosporidium spp e Giardia spp. em carnívoros silvestres procedentes de diferentes municípios do Estado do Pará, abrangendo animais de cativeiro e de vida livre, o que possibilita a geração de informações epidemiológicas que podem contribuir para futuros estudos sobre a saúde única, que abrange meio ambiente, homens e animais, em especial, na região Amazônica.

\section{Material e métodos}

Aprovado pelo SISBIO, licença N $N^{\circ} 39285$, e pelo Comitê de Ética do Uso de Animais, protocolo de autorização Nº 034/2014 CEUA - 23084.022512/2014-18 (UFRA).

Foram coletadas e examinadas 37 amostras fecais de 13 espécies de carnívoros silvestres de diferentes regiões do Estado do Pará, Amazônia, Brasil, sendo quatro de vida livre e 33 de cativeiros. Nove animais foram classificados como jovens (idade inferior a 12 meses) e 28 como adultos (mais de 12 meses de idade), totalizando 15 fêmeas e 22 machos.

As espécies dos carnívoros silvestres do presente estudo foram identificadas por observações morfológicas realizadas pelos médicos veterinários dos locais das coletas a partir da comparação das características da espécie descritas por Reis et al. (2010).

Foram analisadas amostras fecais de quatro cachorro-do-mato (Cerdocyon thous) de vida livre, os demais animais são cativos: 13 jaquatiricas (Leopardus pardalis), três sussuaranas (Puma concolor), três furões (Galictis cf. vittata), três onças pintada (Panthera onça), três juparás (Potos flavus), duas lontras (Lutra longicaudis), uma irara (Eira barbara), um furão grande (Galictis vittata), um gato do mato (Leopardus tigrinus), uma ariranha (Pteronura brasiliensis), um gato-morisco (Puma vagouaroundi) e um cachorro vinagre (Speothos vanticus).

As coletas foram realizadas entre março de 2015 e fevereiro de 2017. As amostras de animais cativos procederam de sete mantenedoras de animais silvestres localizadas nos municípios de Belém, Santarém, Capitão Poço e Terra Alta e foram feitas nos recintos nos quais os animais estavam alojados. Todos os animais foram deixados em gaiolas individuais que foram previamente higienizadas com o objetivo de evitar contaminação ambiental do material coletado. Preconizou-se recolher as amostras imediatamente após defecação, sem a necessidade de contenção dos animais, obtendo-se dados referentes ao sexo, faixa etária, procedência e local dos mesmos. As amostras foram acondicionadas em frasco coletor universal estéril sem presença de qualquer tipo de conservantes, e mantidas sob refrigeração até as análises necessárias. Já as amostras de animais de vida livre, procederam de animais mortos e que foram obtidas durante necropsias, com a retirada do material fecal diretamente do trato digestivo inferior dos animais, realizadas no Laboratório de Patologia Animal da Universidade Federal Rural da Amazônia (LABOPAT/UFRA). 
As amostras foram processadas e analisadas em um período de até 24 horas no Laboratório de Protozoários Intestinais da Seção de Parasitologia do Instituto Evandro Chagas/Ministério da Saúde, Ananindeua, Pará. Durante o processamento foi priorizada a utilização da porção central do bolo fecal, por apresentar menor exposição a contaminantes ambientais que poderiam influenciar nos resultados das análises.

Para pesquisa de cistos de Giardia spp. e oocistos de Cryptosporidium spp. foram utilizados os métodos direto com lugol e coloração de Kinyoun (Ziehl-Neelsen modificado-ZNm), respectivamente. Foram confeccionadas duas lâminas por amostra e analisadas sob microscopia de luz em objetivas de 40x para a observação dos cistos de Giardia spp. e 100x para oocisto de Cryptosporidium spp.

Para a realização do exame direto, utilizou-se uma pequena alíquota do material fecal, retirada de diferentes partes do bolo fecal, misturada a quatro a cinco gotas de lugol em uma lâmina de vidro, numerada e coberta com lamínula $24 \mathrm{~mm} \times 32 \mathrm{~mm}$, seguida de observação ao microscópio.

Na técnica de Ziehl-Neelsen modificado, utilizou-se dois gramas de fezes. O conteúdo foi diluído e homogeneizado em um tubo tipo falcon contendo $10 \mathrm{~mL}$ de formalina tamponada e filtrada em gaze dobrada para a retirada de resíduos grosseiros. Após esta etapa, cerca de $4 \mathrm{~mL}$ de éter foi acrescido ao filtrado para centrifugação (2.000 rpm x 5 minutos). Uma gota do sedimento foi utilizada na confecção do esfregaço, corada com fucsina carbólica e observada ao microscópio.

Foi também empregado um método imunológico comercial RIDA ${ }^{\circledR}$ QUICK Cryptosporidium/Giardia/Entamoeba Combi (N1722), que detecta os antígenos dos parasitas simultaneamente. Os elevados índices de sensibilidade e especificidade do método imunológico utilizado (acima de 90,0\%) fazem do método uma ferramenta complementar ao diagnóstico microscópico, aumentando a probabilidade de detecção destes parasitas.

A realização do teste imunocromatográfico obedeceu ao protocolo do fabricante. De forma resumida, foi feito um homogeneizado utilizando $50 \mathrm{mg}$ da amostra diluída em um $\mathrm{mL}$ de tampão de extração. Uma alíquota de $0,5 \mathrm{~mL}$ do sobrenadante obtido foi colocada em contato com as tiras contendo os anticorpos, procedendo-se a leitura após cinco minutos.

Estatisticamente os dados foram analisados utilizando-se frequências absolutas e relativas pelo programa Bioestat 5.0.

\section{Resultados e discussão}

Das 37 amostras de fezes analisadas, 24,32\% (9/37) foram positivas, correspondendo a 5,4\% (2/37) para Cryptosporidium spp. e 18,91\% (7/37) para Giardia spp., respectivamente, ao qual nenhum animal apresentou infecção concomitante para os agentes.

As amostras positivas para Cryptosporidium spp., foram obtidas de dois animais adultos, sendo uma fêmea e um macho, correspondendo a uma jaguatirica ( $L$. pardalis) de cativeiro (município de Capitão Poço) e um cachorro-do-mato (C. thous) de vida livre necropsiado (município de Belém). As amostras foram positivas para Cryptosporidium spp. no método de ZNm. Todavia, apenas a amostra de $C$. thous foi positiva no teste imunológico, sugerindo-se que o oocisto de Cryptosporidium observado na amostra da jaguatitica não pertença a C. parvum ao qual o teste imunocromatográfico é indicado, e sim a outra espécie deste gênero.

A realização de genotipagem com marcadore moleculares tem auxiliado na identificação desses parasitos, como no trabalho de Carmena et al (2017) que poderam identificar em carnivoros as espécies G. duodenalis, C. felis, C. hominis, C. Ubiquitum. Informações sobre genotipagem desses protozoários em animais da ordem Carnivora ainda são excassos (RYAN ET AL. 2014), e os achados imunológicos do presente trabalho alerta a presença de Cryptosporidium nesta mastofauna amazônica, tendo a necessidade de futuros estudos moleculares para uma caracterização parasito-hospedeiro.

Diversos autores como Kar et al. (2011) ressaltam que metodologias estão disponíveis para o diagnóstico confirmatório da criptosporidiose, podendo ser estabelecido por meio de exames parasitológicos (sedimentação, flutuação ou a centrífugosedimentação), e técnica de coloração de Ziehl-Neelsen ou Kinyoun, aos quais foram utilizados na presente pesquisa (XIAO; CAMA, 2006). Tais afirmações evidenciam que outros métodos de diagnósticos aplicados ao agente auxiliam cada vez mais o diagnóstico, o que inevitavelmente contribui para um melhor conhecimento da epidemiologia do Cryptosporidium spp. no Brasil e no mundo.

No Brasil há poucas informações sobre a ocorrência de Cryptosporidium em espécies de carnívoros silvestres, dentre essas, Fanfa et al. (2011), utilizando diferentes métodos parasitológicos, relataram a ocorrência de Cryptosporidium spp. em um Puma concolor (puma), adulto, assintomático, mantido em um criatório conservacionista em Santa Maria, Rio Grande do Sul. Em outro estudo, Holsback et al. (2013), analisaram amostras fecais de 14 carnívoros silvestres, de seis espécies diferentes e, observaram, positividade para oocistos de Cryptosporidium spp. em duas espécimes de L. tigrinus (gato do mato) de centros de reabilitação dos Estados do Mato Grosso do Sul e São Paulo. Resultado semelhante aos obtidos nessa pesquisa no Estado do Pará, que em 37 animais de 13 diferentes espécies, diagnosticou-se Cryptosporidium spp. em dois espécimes, sendo uma jaguatirica e um cachorro do mato.

Em outros países, Pezzani et al. (2001) relataram o primeiro registro de Cryptosporidium spp. em $P$. concolor mantido em cativeiro na Argentina. Na Irlanda Stuart et al. (2013) utilizaram técnica molecular e detectaram a presença de Cryptosporidium em 5\% (4/81) de indivíduos da espécies Mustela vison, uma espécie carnívora de hábitos aquáticos. Uma frequência semelhante a registrada no presente estudo. Dentre as amostras identificadas, três foram caracterizadas como C. andersoni, reforçando a necessidade de estudos moleculares para a elucidação da espécie de Cryptosporidium identificada através de microscopia neste estudo. Nas demais espécies estudadas na Irlanda, a maioria das quais de ambiente predominantemente terrestre, nenhuma amostra foi positiva para o agente.

Vale ainda destacar que nem todos os animais que são diagnosticados com a infecção por Cryptosporidium spp. possuem manifestações clínicas de doença, atuando assintomaticamente como fontes de infecção desse agente, albergando-o em seu trato intestinal e eliminando grandes quantidades de oocistos viáveis para o ambiente (BOYER; KUCZYNSKA, 2010). 
Com relação aos resultados da pesquisa de Giardia spp., a positividade detectada em sete indivíduos (18,91\%), ocorreu em três animais jovens e quatro adultos, sendo quatro fêmeas e três machos, procedentes de criatórios dos municípios de Belém e Capitão Poço. Como todas as amostras foram positivas para Giardia spp. tanto no método direto como no imunológico, provavelmente, pertencem à espécie G. lamblia, ao qual o teste imunocromatográfico utilizado é indicado. A G. lamblia, G.duodenalis, G. intestinalis são sinômimos, outras espécies de Giardia spp. são encontradas em outros hospedeiros (roedores, aves e anfíbios), e a proximidade da inter-relação entre homens e animais, pode ser um determinante em potencial zoonótico para essa parasitoses (SANTARÉM et al., 2004). $G$. duoedanalis pode parasitar o homem, outros mamíferos e mamíferos silvestres, a espécie é dividida em 8 genótipos (A-G) sendo os genótipos A e B mais comum no homem, os demais genótipos apresentam caráter zoonótico muito frenquente em amíferos (FENG; XIAO, 2011).

No presente estudo, cinco das 13 espécies de carnívoros silvestres, estavam parasitadas, com sete animais positivos para Giardia spp., ocorrendo em três $L$. pardalis (jaguatiricas), e em um P. brasiliensis (ariranha), F. concolor (sussuarana), L. longicaudis (lontra) e C. thous (cachorro do mato), respectivamente. Dos animais, o cachorro do mato era de vida livre e a Puma concolor foi a única a apresentar diarreia.

No Brasil, Farret et al. (2008), analisaram amostras de fezes de 14 carnívoros silvestres mantidos em cativeiro em criadouro conservacionista no Estado do Rio Grande do Sul. Entre as espécies pesquisadas, incluíram-se cinco gatos do mato pequeno (Leopardus tigrinus), três quatis (Nasua nasua), três graxains do campo (Lycalopex gymnocercus) e três guaxinins (Procyon lotor). Os autores verificaram que L. tigrinus foi à única espécie que apresentou infecção elevada para Giardia spp. Infecção mista por cistos de Giardia spp., oocistos de Cryptosporidium spp. e Cystoisopora sp. estava presente em $N$. nasua, a espécie L. gymnocercus estava parasitada por oocistos de Cystoisospora sp. e P. lotor por cistos de Giardia spp. e oocistos de Cryptosporidium spp. Os animais não apresentavam sinais clínicos das parasitoses, e os autores concluíram que estes eram portadores assintomáticos e atuavam como propagadores destes parasitos para o ambiente. O mesmo pode ser considerado na presente pesquisa, já que apenas um animal apresentou sinais clínicos de diarreia.

Recentemente, Borges et al. (2017), utilizando métodos parasitológicos (Kinyoume parasitológico (centrífugo flutuação em solução de sulfato de zinco), analisaram 337 amostras fecais de carnívoros silvestres coletadas nos Estados do Amapá, Amazonas, Pará e Rondônia, correspondendo, a 313 Lontra longicaudis (Iontra tropical) e 24 Pteronura brasiliensis (ariranha). A frequência de positividade de infecção para pelo menos um patógeno foi de $27 \%$ (94/337), ao qual a infecção por Cryptosporidium spp. foi maior que Giardia spp. Já coinfecção por ambos os protozoários foi de 4,47\% (14/313) nas amostras de L. longicaudis e 20,83\% (5/24) nas de P.brasiliensis.

Os resultados encontrados por Borges et al. (2017), assemelhamse aos encontrados no presente estudo em relação à positividade para a Giardia spp., ao qual, evidenciou-se resultado positivo para a $P$. brasiliensis e para a $L$. longicaudis tanto no método direto quando no imunocromatográfico, acreditando-se portanto, que tais espécies podem ser mantenedoras e disseminanadoras de tal agente para o meio ambiente e para outros animais. Todavia, para Cryptosporidium spp., na presente pesquisa, o resultado foi negativo em ambas as espécies, mesmo com a alta sensibilidade do método imunológico utilizado.

Na Espanha, Méndez-Hermida et al. (2007), pesquisaram por imunofluorescência direta, 437 amostras fecais de Lutra lutra (lontra selvagem) coletadas de 161 localidades. Foram detectados oocistos de Cryptosporidium spp. em 3,9\% (17/437) e cistos de Giardia spp. em 6,8\% (30/437) das amostras. Os resultadosb do presente trabalho demonstram que as lontras podem contribuir para a contaminação dos cursos de água, embora sejam necessários mais estudos para determinar quais espécies ou genótipos de Cryptosporidium e Giardia infectam esses animais.

Na Croácia foi realizado uma pesquisa de Giardia spp. onde se utilizou microscopia de luaz e imunofluorescência, ao qual foram analisadas 131 amostras fecais de 57 espécies de mamíferos cativos no zoológico de Zagreb. A prevalência geral foi de $29 \%$ (38/131), considerada alta pelos autores, sendo que todos os animais apresentavam-se assintomáticos no momento da coleta. Com relação aos carnívoros presentes no zoológico de Zagred, foram coletadas 21 amostras e destas, $12(57,1 \%)$ mostraram-se positivas para Giardia spp. (BECK et al., 2011).

Leopardus pardalis foi a espécie que apresentou maior positividade para os agentes estudados na presente pesquisa, correspondendo a $44,44 \%$ (4/9) dos animais parasitados. Das 13 amostras coletadas de jaguatirica, quatro mostraram-se parasitadas, todas procedentes de animais de cativeiros, porém pouco se sabe sobre esses agentes nas espécies aqui estudadas, apesar da abundante literatura a respeito da ocorrência em hospedeiros humanos e animais domésticos, poucos são os estudos sobre o papel dos animais silvestres na manutenção e disseminação desses agentes (THOMPSON et al., 2010).

$\mathrm{Na}$ presente pesquisa foi possível observar que os agentes pesquisados ocorrem em carnívoros silvestres mantidos em cativeiros no Estado do Pará, sendo estes dados importantes para um melhor entendimento do problema em relação à epidemiologia parasitária ambiental envolvendo diferentes espécies de animais silvestres que se tornam potenciais vetores desses importantes parasitas. Considera-se oportuno referenciar o estudo de FARRET et al. (2008), em carnívoros silvestres mantidos em cativeiro no sul do Brasil, o qual relata o parasitismo por protozoários gastrintestinais com potencial zoonótico, destacando que a situação de cativeiro torna os animais mais susceptíveis às doenças pelo estresse e a concentração destes em um mesmo local, o que pode gerar problemas de saúde nestes animais, inclusive em animais domésticos e/ou no homem, visto que que esses podem albergar parasitos zoonóticos.

Adicionalmente, é notória a ocorrência de Cryptosporidium e Giardia spp. em carnívoros silvestres de vida livre e cativos em diferentes regiões do mundo (APPELBEE et al., 2005), com prevalências que chegam a atingir valores acima de $50 \%$. De acordo com os resultados obtidos neste estudo é possível concluir que a circulação destes agentes nos animais de ambientes florestais amazônicos sejam tão comum quanto nas demais regiões do Brasil e do mundo. Assim, novos estudos 
em animais silvestres de diferentes espécies irão contribuir para a uma melhor compreensão acerca da epidemiologia destes agentes na região Amazônica e no Brasil. Ademais, a circulação destes agentes em animais de cativeiro, favorecendo a manutenção e a disseminação dos mesmos nestes ambientes, mostra a da necessidade de avaliações periódicas bem como a identificação das prováveis fontes de infecção para os animais silvestres.

\section{Referências}

AIRES, W. O., FRIAS, R. B., OLIVEIRA, L. R., \& PIRES, F. A. (2008). Principais parasitas de felinos selvagens. Revista Científica Eletrônica de Medicina Veterinária. Ano VI, 11, 1-5.

APPELBEE, A. J., THOMPSON, R. C., \& OLSON, M. E. (2005). Giardia and Cryptosporidium in mammalian wildlife - Current status and future needs. Trends in Parasitology, 21(8), 370-376.

BARR, S. C., \& BOWMAN, D. D. (2010). Doenças infecciosas e parasitárias em cães e gatos - consulta em 5 minutos. 1. ed. São Paulo: Revinter, 640p.

BECK, R., SRONG, H., BATA, I., LUCINGER, S., POZIO, E., \& CACCIÓ, S. M. (2011). Prevalence and molecular typing of Giardia spp. in captive mammals at the zoo of Zagreb, Croatia. Veterinary Parasitology, 175(1-2), 40-46.

BORGES, J. C. G., LIMA, D. S., CALERA, B. M., \& MARMONTEL, M. (2017) Cryptosporidium spp. and Giardia sp. in Neotropical river otters (Lontra longicaudis) and giant otters (Pteronura brasiliensis) in northern Brazil. Journal of the Marine Biological Association of the United Kingdom, 1-5.

BOYER, D.G., \& KUCZYNSKA, E. (2010). Prevalence and concentration of Cryptosporidium oocysts in beef cattlenpaddock soils and forage. Foodborn Pathogens and Disease, 7(8), 893900,

BRIGGS, A. D., BOXALL, N. S., VAN SANTEN, D., CHALMERS, R. M., \& MCCARTHY, N. D. (2014). Approaches to the detection of very small, common, and easily missed outbreaks that together contribute substantially to human Cryptosporidium infection. Epidemiology Infectolology, 142(9), 1869-76.

FANFA, V. R., FARRET, M., SILVA, A. S., \& MONTEIRO, S. (2011). Endoparasitoses em puma (Puma concolor) na Regiao Sul do Brasil [Endoparasitoses in cougar (Puma concolor) from the Southern region of Brazil] Departamento de Microbiologia e Parasitologia da Universidade Federal de Santa Maria, RS, Brasil. Acta Veterinaria Brasilica, 5(1), 100-102.

FARRET, M. H., FANFA, V. R., SILVA, A. S., ZANETTE, R. A., \& MONTEIRO, S.G. (2008). Parasitismo por protozoários gastrointestinais em carnívoros silvestres mantidos em cativeiro no sul do Brasil. Revista Portuguesa de Ciências Veterinárias, 103 (565-566), 93-95.

FENG, Y., XIAO, L. (2011). Zoonotic Potential and Molecular Epidemiology of Giardia Species and Giardiasis Clinical Microbiology Reviews, 24 (1), 110-140.

FIORELLO, C. V., ROBBINS, R. G., MAFFEI, L., \& WADE, S. E. (2005). Parasites of free-ranging small canids and felids in the Bolivian Chaco. Journal of Zoo and Wildlife Medicine, 37(2), 130134.

\section{Conclusão}

A pesquisa comprova a presença dos agentes Cryptosporidium spp. e Giardia spp. em carnívoros silvestres tanto mantidos em criatórios como nos de vida livre no Estado do Pará, o que possibilita considerar que esses animais podem atuar como fontes de infecção para outros animais e para o homem.

HOLSBACK, L., CARDOSO, M. J. L., FAGNANI, R., \& PATELLI, T. H. C. (2013). Natural infection by endoparasites among free-living wild animals. Infecção natural por endoparasitas em animais silvestres de vida-livre. Revista Brasileira de Parasitologia Veterinária, 22(2), 302-306.

KAR, S., GAWLOWSKA, S., DAUQSCHIES, A., \& BANQOURA, B. (2011). Quantitative comparison of different purification and detection methods for Cryptosporidium parvum oocysts. Journal of Veterinary Parasitology, 177(3-4), 366-370.

LALLO, M. A., RODRIGUES, L. C. S., \& BONDAN, D. F. (2003). Giardíase em cães e gatos- revisão. Revista Clínica Veterinária, 43, 40-44.

LALLO, M. A., ARAÚJO, A. P. R., FAVORITO, S. E., BERTOLLA, P., \& BONDAN, E. F. (2009). Ocorrência de Giardia,

Crytosporidium e microsporídios em animais silvestres em áreas de desmatamentos no Estado de São Paulo, Brasil. Ciência Rural, 39(5), 1465-1470.

LINDSAY, D. S., \& ZAJAC, A. M. (2009). The Biology and Control of Giardia spp and Tritrichomonas foetus. Veterinary Clinics of North America: Small Animal Practice, 39(6), 993-1007.

MARVULO, M. F. V. (2007). Zoonoses. In: CUBAS, Z. S., DIAS, J. L. C., \& SILVA, J. C. R. Tratado de Animais Selvagens. São Paulo: Roca, 1250-1256.

MATEO, M., DE MINGO, M. H., DE LUCIO, A., MORALES, L., BALSEIRO, A., ESPÍ, A., BARRAL, M., LIMA BARBERO, J. F., HABELA, M. A., FERNANDEZ-GARCÍA, J. L., BERNAL, R. C., KOSTER, P. C., CARDONA, G. A., CARMENA, D. Occurrence and molecular genotyping of Giardia duodenalis and Cryptosporidium spp. in wild mesocarnivores in Spain. (2017). Veterinary Parasitology, 235, 86-93

MÉNDEZ-HERMIDA, F., GÓMEZ-COUSO, H., ROMEROSUANCES, R., \& ARES-MAZÁS, E. (2007). Cryptosporidium and Giardia in wild otters (Lutra lutra). Veterinary Parasitology, 144(12), 153-156.

ONICHANDRAN, S., KUMAR, T., SALIBAY, C. C., DUNGCA, J. Z., TABO, H., A. L., TABO, N., TIAN-CHYE, T., LIM, Y. A. L., SAWANGJARIOEN, N., PHIRIYASAMITH, S., ANDIAPPAN, H., ITHOI, I., LAU, Y. L., \& NISSAPATORN, V. (2014). Waterborne parasites: a current status from the Philippines. Parasites \& Vectors, 28(7), 244-251.

PEZZANI, B. C., APEZTEGUÍA, M., BAUTISTA, L. E., \& CASUALDO, J. A. (2001). Prevalence of Cryptosporidium spp. in mammals of La Plata and its rural areas. Acta Bioquímica Clínica Latinoamericana, 35(4), 521-526.

REIS, N. R., PERACCHI, A. L., FREGONEZI, M. N., \& ROSSANEIS, B. K. (2010). Mamíferos do Brasil: guia de identificação. Ed. $1^{\circ}$. Technical Books Editora. p.490. 
RYAN, U., FAYER, R., XIAO, L. (2014). Cryptosporidium species in humans and animals: current understanding and research needs. Parasitology, 141, 1667-1685.

SANTARÉM, V. A.; GIUFFRIDA, R.; ZANIN, G. A. (2004). Cutaneous larva migrans: reports of pediatric cases and contamination by Ancylostomaspp larvae in public parks in Taciba, São Paulo State. Revista da Sociedade Brasileira de Medicina Tropical, 37 (2), 179-181.

SOARES, J. F., SILVA, A. S., OLIVEIRA, C. B., SILVA, M. K., MARISCANO, G., SALOMÃO, E. L., \& MONTEIRO, S. G. (2008). Parasitismo por Giardia sp. e Cryptosporidium sp. em Coendou villosus. Ciência Rural, 38(2), 23-24.

STUART, P., GOLDEN, O., ZINTL, A., DE WAAL, T., MULCAHY, G., MCCARTHY, E., \& LAWTON, C. (2013). A coprological survey of parasites of wild carnivores in Ireland. Parasitology Research Founded as Zeitschrift für Parasitenkunde, 112(10), 3587-3593.
THOMPSON, R. C. A. et al. Parasites emerging disease and wildlife conservation. International Journal for Parasitology, 40, n.10, p. 1163-1170, 2010.

WHITEMAN, C. W., PALHA, M. D. C., MATUSHIMA, E. R., SILVA, A. S. L., \& MONTEIRO, V. C. (2008). Interface entre carnívoros domésticos e silvestres em área de proteção ambiental na amazônia brasileira: indicadores e implicações para conservação. Natureza \& Conservação, 6(1), 174-182.

XIAO, L., \& CAMA, V.( 2006). Cryptosporidium and Cryptosporidiosis In: ORTEGA, Y. R. Foodborne Parasites. Springer: US, 108p.

XIAO, L., \& FAYER, R. (2008). Molecular characterisation of species and genotypes of Cryptosporidium and Giardia and assessment of zoonotic transmission. International Journal Parasitology, Oxiford, 38(11), 1239-1255. 